\title{
Time-Resolved Detection and Analysis of Single Nanoparticle Elec- trocatalytic Impacts
}

\author{
Minkyung Kang, ${ }^{\dagger}$ David Perry, ${ }^{\dagger}, \ddagger$ Yang-Rae Kim, ${ }^{\dagger}$ Alex W. Colburn, ${ }^{\dagger}$ Robert A. Lazenby, ${ }^{\dagger}$ and \\ Patrick R. Unwin*† \\ †Department of Chemistry and ${ }^{\ddagger}$ MOAC Doctoral Training Centre, University of Warwick, Cov- \\ entry, CV4 7AL, UK
}

\section{Supporting Information Placeholder}

\begin{abstract}
There is considerable interest in understanding the interaction and activity of single entities, such as (electro)catalytic nanoparticles (NPs), with (electrode) surfaces. Through the use of a high bandwidth, high signal/noise measurement system, NP impacts on an electrode surface are detected and analyzed in unprecedented detail, revealing considerable new mechanistic information on the process. Taking the electrocatalytic oxidation of $\mathrm{H}_{2} \mathrm{O}_{2}$ at ruthenium oxide $\left(\mathrm{RuO}_{\mathrm{x}}\right)$ NPs as an example, the rise time of currenttime transients for NP impacts is consistent with a hydrodynamic trapping model for the arrival of a NP with a distancedependent NP diffusion-coefficient. NP release from the electrode appears to be aided by propulsion from the electrocatalytic reaction at the NP. High frequency NP impacts, orders of magnitude larger than can be accounted for by a single pass diffusive flux of NPs, are observed that indicate the repetitive trapping and release of an individual NP that has not previously recognized. The experiments and models described could readily be applied to other systems and serve as a powerful platform for detailed analysis of NP impacts.
\end{abstract}

An important frontier in electrochemistry is measuring the behavior of individual nano-entities such as nanoparticles (NPs), nanowires and nanorods and relating this to other properties such as size, structure and electronic characteristics, so as to develop fundamental understanding and rational applications. ${ }^{1-3}$ An interesting approach for observing the electrochemical properties of catalytic NPs is to monitor their impact (or landing) from solution onto a collector electrode, as introduced by Bard et al.,4,5 and developed by several groups. ${ }^{6-12}$ In order to resolve such impacts, the use of a small-sized ultramicroelectrode (UME) is mandatory to reduce both background currents and the impact frequency. To enhance the impact signal to background current, electrode surfaces have been modified with $\mathrm{Hg}$ or $\mathrm{Bi}^{7}$ and borondoped diamond ${ }^{12}$ has also been used as an UME material. Alternatively, scanning electrochemical cell microscopy (SECCM) functioning as an ultramicro-electrochemical cell system offers particularly low background currents by reducing the area of the collector electrode, as well as offering the widest range of support electrodes. This is because the electrochemical cell is formed by meniscus confinement, rather than electrode encapsulation (Figure 1). ${ }^{13}$ Despite these innovations, detailed analysis of the form of the current-time profile which is the primary signal for the landing (and detachment) of a single NP on an electrode has not yet been forthcoming, but would represent a huge advance towards understanding the impact process. Herein, we are able to analyze this process as never before and deduce key information on the NP arrival and release process from individual impact transients. Moreover, we show that impact frequencies can be orders of magnitude higher than expected based on single pass diffusion due to the repetitive impact and release of a single NP.
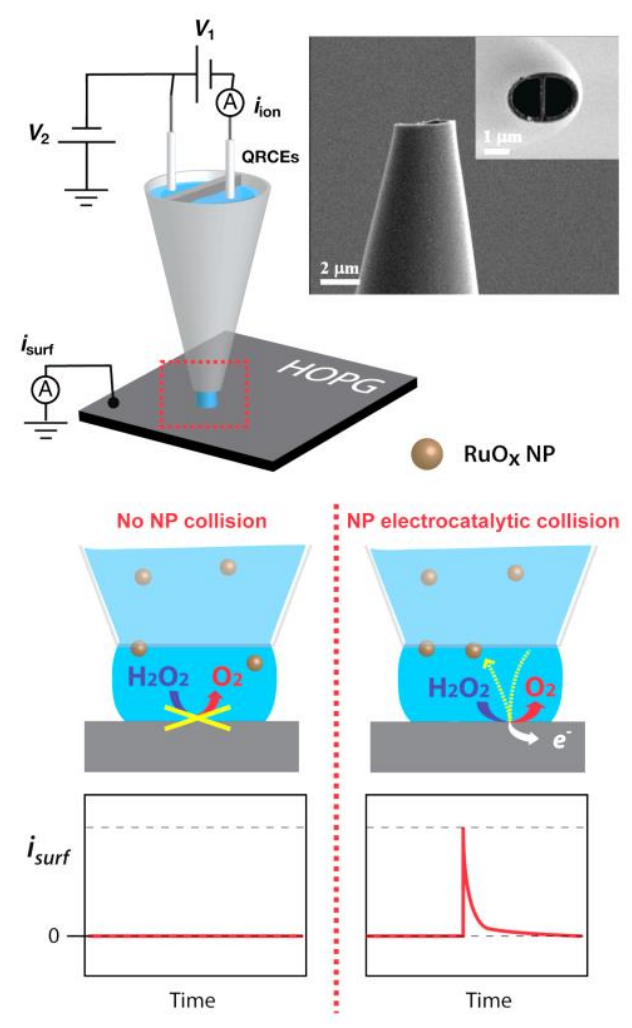
Figure 1. $\mathrm{RuO}_{\mathrm{x}} \mathrm{NP}$ landing experiments in an ultramicroelectrochemical cell, showing the cell set up (top), with a typical theta pipette for meniscus contact and NP delivery to a working electrode (HOPG) substrate. There is no oxidation of $\mathrm{H}_{2} \mathrm{O}_{2}$ at the HOPG electrode surface, i.e. no surface current ( $i_{\text {surf }}$ ), as shown on the bottom left, unless a NP impacts with the surface and sets off the electrocatalytic oxidation of $\mathrm{H}_{2} \mathrm{O}_{2}$ at the NP (bottom right).

In this paper we use SECCM ${ }^{13}$ to investigate $\mathrm{H}_{2} \mathrm{O}_{2}$ oxidation at ruthenium oxide $\left(\mathrm{RuO}_{\mathrm{x}}\right) \mathrm{NPs}$, determining the NP landing characteristics and the distribution of kinetics currents for individual impacts within an ensemble of colliding NPs, with unprecedented time resolution. The heterogeneous kinetics of $\mathrm{H}_{2} \mathrm{O}_{2}$ electro-oxidation has been studied extensively at a variety of nanomaterials, ${ }^{13}$ among which several metal oxides appear to be promising, particularly for bioanalytical applications, due to the biocompatibility and robust electrocatalytic performance. ${ }^{14-17} \mathrm{RuO}_{\mathrm{x}}$ is especially interesting as it catalyzes $\mathrm{H}_{2} \mathrm{O}_{2}$ electro-oxidation at relatively low overpotentials in physiological environments. ${ }^{15,16}$ The experiments reported herein allow us to measure the residence time and interaction of $\mathrm{RuO}_{x} \mathrm{NPs}$ with an electrode during electroctalysis.

The ultramicro-electrochemical cell was made by meniscus contact of a highly oriented pyrolytic graphite (HOPG) collector electrode from a tapered dual-barrelled borosilicate theta pipette (end diameter $3 \mu \mathrm{m}$ ), filled with a solution of $\mathrm{RuO}_{\mathrm{x}} \mathrm{NPs}$ and $0.5 \mathrm{mM} \mathrm{H}_{2} \mathrm{O}_{2}$ in $0.1 \mathrm{M}$ phosphate buffer $(\mathrm{pH}$ 7.4) and containing a $\mathrm{Ag} / \mathrm{AgCl}$ quasi-reference counter electrode (QRCE) in each channel (Figure 1). ${ }^{13}$ The pipette was approached towards the HOPG with a z-piezoelectric positioner while monitoring the ion-conductance current between the barrels $\left(i_{\text {ion }}\right)$ with a potential bias of $0.1 \mathrm{~V}$ between the two $\mathrm{Ag} / \mathrm{AgCl}$ QRCEs $\left(V_{1}\right)$. Once the meniscus was in contact with the HOPG, sensed as an abrupt change in $i_{\text {ion, }}{ }^{13} V_{1}$ was set to o $\mathrm{V}$ and current-time $(i-t)$ traces were recorded from the HOPG substrate $\left(i_{\text {surf }}\right)$. Further details of the instruments and materials used can be found in the Supporting Information (S1.1). HOPG was selected as the collector electrode as it exhibits exceptionally low background currents, ${ }^{18,19}$ and is relatively insensitive to $\mathrm{H}_{2} \mathrm{O}_{2}$ oxidation over the potential range where $\mathrm{RuO}_{\mathrm{x}}$ is an effective electrocatalyst (Supporting Information, Figure S1). Moreover, many types of NPs exhibit weak interaction with HOPG, ${ }^{20,21}$ and this enabled us to monitor $\mathrm{H}_{2} \mathrm{O}_{2}$ oxidation on the $\mathrm{RuO}_{x}$ NPs with glancing collisions rather than sticking landings where the NP would remain, and accumulate on the support electrode.
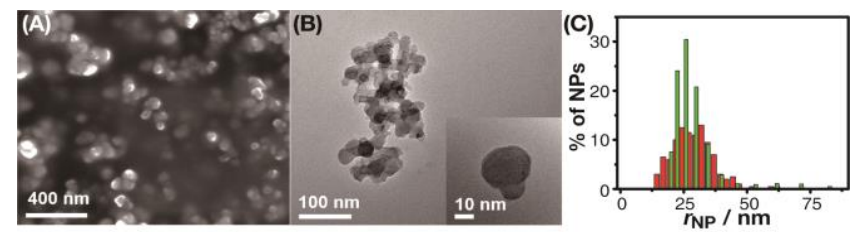

Figure 2. (A) FE-SEM and (B) TEM images of $\mathrm{RuO}_{x}$ NPs synthesized with sodium citrate. (C) Size distribution from the analysis of TEM images (red) and from DLS (green), in terms of the particle radius, $r_{\mathrm{NP}}$.

$\mathrm{RuO}_{\mathrm{x}} \mathrm{NPs}$ were synthesized with sodium citrate (Supporting Information, S1.3) and characterized by field emission- scanning electron microscopy (FE-SEM) and transmission electron microscopy (TEM), Figures $2 \mathrm{~A}$ and $2 \mathrm{~B}$. Sodium citrate was used as a capping agent as it promoted the formation of well-dispersed $\mathrm{RuO}_{\mathrm{x}} \mathrm{NPs}$ with a reasonably regular size and shape (Figure 2), but would not lead to much inhibition of electron-transfer in collision experiments unlike some alternative organic capping agents. ${ }^{22}$ The apparent NP radius, $r_{\mathrm{NP}}$, was estimated from the analysis of TEM images of NPs, with a mean value of $28 \pm 8 \mathrm{~nm}(N=200)$, and from dynamic light scattering (DLS) for the same solution conditions as for the electrochemical measurements $(26 \pm 5 \mathrm{~nm})$, with the results of both analysis shown in Figure $2 \mathrm{C}$. Details of DLS are given in Supporting Information (S1.3). The capping step also enhanced the colloidal stability of $\mathrm{RuO}_{\mathrm{x}} \mathrm{NPs}$ in aqueous solution, as a result of a larger absolute $\zeta$-potential value than without capping agent (Supporting Information, Table $\mathrm{S} 1) .{ }^{23} \mathrm{RuO}_{\mathrm{x}} \mathrm{NPs}$ synthesized without sodium citrate, in contrast, showed uneven structures with a broad distribution of both apparent size (Supporting Information, Figure S2) and the current signal in landing experiments, due to a predominance of agglomerates (Supporting Information, Figure S3).
(A)

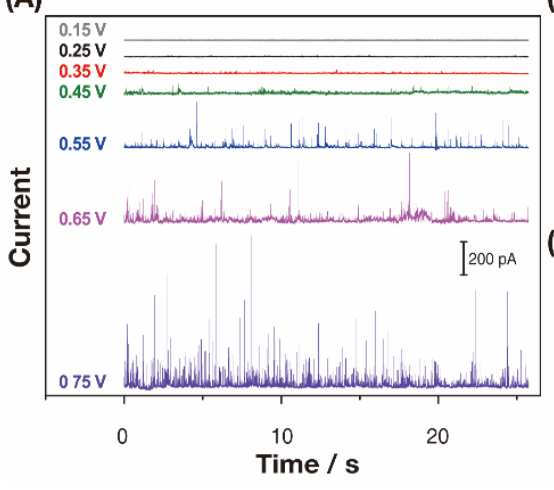

(B)

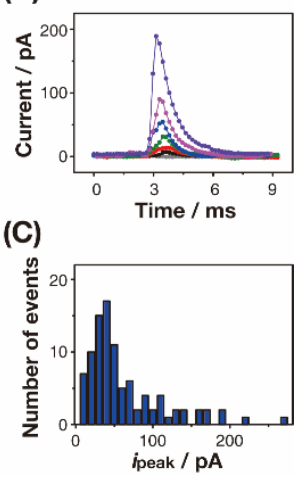

Figure 3. (A) Current $\left(i_{\text {surf }}\right)$ responses for o.5 $\mathrm{mM} \mathrm{H}_{2} \mathrm{O}_{2}$ oxidation with $15 \mathrm{pM} \mathrm{RuO}_{\mathrm{x}} \mathrm{NPs}$ in o.1 M phosphate buffer solution ( $\mathrm{pH} 7.4)$ at different $E_{\text {app }}$ at the HOPG collector electrode $(0.15 \mathrm{~V}, 0.25 \mathrm{~V}, 0.35 \mathrm{~V}, 0.45 \mathrm{~V}, 0.55 \mathrm{~V}, 0.65 \mathrm{~V}$ and $0.75 \mathrm{~V}$ vs. $\mathrm{Ag} / \mathrm{AgCl} \mathrm{QRCE})$. (B) Example current responses of individual impacts of $\mathrm{RuO}_{\mathrm{x}} \mathrm{NPs}$ at the different $E_{\text {app }}$ with the color matched with (A); the bigger the current magnitude the higher the $E_{\text {app }}$ (C) Distribution of peak currents, $i_{\text {peak }}$, from collision experiments at $0.55 \mathrm{~V}$.

$\mathrm{RuO}_{\mathrm{x}} \mathrm{NP}$ impacts with the collector electrode were observed only in the presence of $0.5 \mathrm{mM} \mathrm{H}_{2} \mathrm{O}_{2}$ in $0.1 \mathrm{M}$ phosphate buffer solution ( $\mathrm{pH}$ 7.4) due to $\mathrm{H}_{2} \mathrm{O}_{2}$ oxidation on $\mathrm{Ru}$ $\mathrm{O}_{\mathrm{x}}$ NPs when they made the contact with the HOPG support (Figure 3). These data are representative of more than $8 \mathrm{ex}-$ perimental runs carried out on this system. Control measurements, with and without $\mathrm{H}_{2} \mathrm{O}_{2}$ present (at a collector electrode potential of $0.55 \mathrm{~V}$ ), are given in Supporting Information, Figure $\mathrm{S}_{4}$. Results at different applied potentials $\left(E_{\text {app }}\right)(0.15,0.25,0.35,0.45,0.55,0.65$ and $0.75 \mathrm{~V})$ showed that in the presence of $\mathrm{H}_{2} \mathrm{O}_{2}$ distinct features in the $i-t$ trace started to appear at $0.25 \mathrm{~V}$ (Figure $3 \mathrm{~A}$ ). The individual $i-t$ response shape (Figure ${ }_{3} \mathrm{~B}$ ) was characterized by a fast rise to a peak $\left(i_{\text {peak }}\right)$ and a slower decay back to the baseline, within 3 ms, during the single NP impacts on the collector electrode. $i_{\text {peak }}$ tended to increase with more positive $E_{\text {app. }}$ At $0.25 \mathrm{~V}$, events with $i_{\text {peak }}$ of just $7 \pm 1 \mathrm{pA}$ and charge of $11 \pm 4 \mathrm{fC}$ could 
be seen (corresponding to the 2-electron oxidation of just 35 $( \pm 14) \times 10^{3}$ molecules of $\left.\mathrm{H}_{2} \mathrm{O}_{2}\right)$.

A potential of $0.55 \mathrm{~V}$ was chosen as an optimized value for further studies to obtain a sufficiently large current response for $\mathrm{H}_{2} \mathrm{O}_{2}$ electro-oxidation to be made with good signal to noise and bandwidth (Figure $3 \mathrm{~A}$ ), while minimizing side reactions such as water splitting that can occur on $\mathrm{RuO}_{\mathrm{x}} \mathrm{NPs}$ at excessively positive potential (Support Information, Figure $\mathrm{S}_{5}$ ). ${ }^{24}$ The mean value of $i_{\text {peak }}$ at $0.55 \mathrm{~V}$ (Figure ${ }_{3} \mathrm{C}$ ) was $46 \pm 16$ pA corresponding reasonably well to that expected for the diffusion-controlled steady-state current $\left(i_{\mathrm{ss}}\right)$ predicted for a $\mathrm{NP}$ on a surface, based on the NP size distribution (Figure $2 \mathrm{C}): 25,26$

$$
i_{\mathrm{ss}}=4 \pi(\ln 2) n F D_{\mathrm{H}_{2} \mathrm{O}_{2}} C_{\mathrm{H}_{2} \mathrm{O}_{2}} r_{\mathrm{NP}}(1)
$$

where $n$ is the number of electrons transferred per $\mathrm{H}_{2} \mathrm{O}_{2}$ (2), $F$ is the Faraday constant $\left(96485 \mathrm{C} \mathrm{mol}^{-1}\right), \mathrm{D}_{\mathrm{H}_{2} \mathrm{O}_{2}}$ is the diffusion coefficient of $\mathrm{H}_{2} \mathrm{O}_{2}$ in o.1 $\mathrm{M}$ phosphate buffer solution $\left(1.46 \times 10^{-5} \mathrm{~cm}^{2} \mathrm{~s}^{-1}\right),{ }^{27} \mathrm{C}_{\mathrm{H}_{2} \mathrm{O}_{2}}$ is the concentration of $\mathrm{H}_{2} \mathrm{O}_{2}$ (0.5 mM). This simple analysis yields $i_{\mathrm{ss}}=38 \pm 10 \mathrm{pA}$.

The high rates of mass transport to NPs of this size means that the characteristic steady-state diffusion time, $0.5 \mu \mathrm{s}(\approx$ $\left.r_{\mathrm{NP}^{2}} / D_{\mathrm{H}_{2} \mathrm{O}_{2}}\right)$, is rapid and much faster than the response time of the electrochemical measurement system. The electrochemical current at any time (taking account of the instrument response function for the current measurement; Supporting Information S2) is thus determined by the occupancy of the NP with the electrode surface. Since $i_{\text {peak }}$ is close to that expected for a diffusion-limited process (see above), $i_{\text {peak }}$ represents an occupancy of one of the NP with the surface, and $i(t) / i_{\text {peak }}$ is thus the relative occupancy at time, $t$.

(A)

(C)
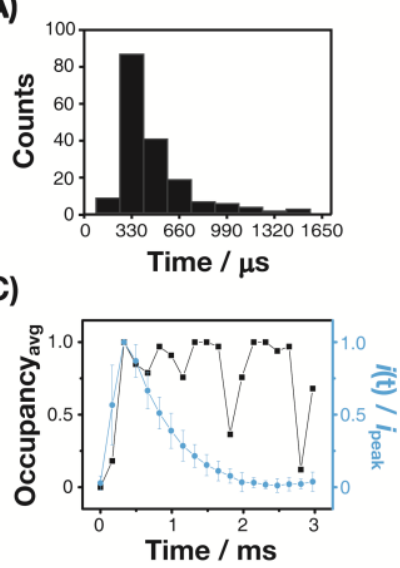

(D)
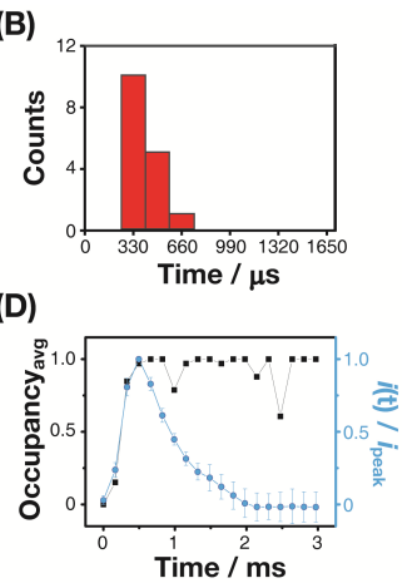

Figure 4. Histograms of the rise time from (A) 200 simulations and (B) 16 experimental transients. Experimental $i-t$ traces (blue lines) are presented in (C) and (D). These are the average (one standard deviation) of: (C) 10 individual transients that had a rise time of $330 \mu$ s and (D) 5 individual traces that had a rise time of $500 \mu \mathrm{s}$. Shown alongside are simulated occupancy traces (black lines), which displayed similar rise time for comparison.

The excellent signal to noise and high bandwidth in our experiment allowed us to examine individual transients in unprecedented detail and compare the results to threedimensional (3D) random walk simulations of NP landing, details of which are presented in Supporting Information, S2. Although the model has been developed for a spherical NP, to which most systems will approximate, it would be possible to consider non-spherical NPs (variable direction-dependent diffusion coefficient). Furthermore, we have shown that NP aggregation is relatively unimportant in this system, but if such effects occurred, they could be incorporated into the model, e.g. through a time-dependent particle size and population, representing the aggregation and de-aggregation kinetics. In brief, for the simulations, we considered an electrolyte zone above the collector electrode of a similar size to the SECCM meniscus. Electron transfer between the electrode and NP was reasonably assumed to occur when the NP was within $1 \mathrm{~nm}$ of the electrode. ${ }^{28}$ We performed 200 simulations each of $3 \mathrm{~ms}$ duration with the NP having the same initial position at the start $(5 \mathrm{~nm}$ above the electrode, over the center). As the NP moved from the start position and began to encounter the electrode, the simulations showed a distribution of rise times, defined as the time taken for the occupancy to change from an average of 0.1 to o.9, centered around $465 \mu \mathrm{s}$, as summarized in Figure $4 \mathrm{~A}$. This distribution is seen to be consistent with the current-time transients observed experimentally (Figure $4 \mathrm{~B}$ ). A key factor determining the rise time is a distance-dependent diffusion coefficient, expected for a spherical particle near a wall in solution, which slows the NP speed of motion, the closer the NP moves to the electrode, leading to hydrodynamic trapping (see Supporting Information, section S2).

In fact, the model simulations predict the NP to remain, on average, near the electrode surface due to the hindered diffusion of the NP, resulting in a occupancy close to 1 for an extended period. ${ }^{29}$ However, in the experiments, after reaching a peak, the current shows a decay with time over a few ms. This difference in behavior between the experiment and model simulation can be seen in Figures ${ }_{4} \mathrm{C}$ and $\mathrm{D}$ which each show the occupancy extracted from single example simulation runs plotted with current-time profiles obtained experimentally, as the average of 10 transients $(C)$ and 5 transients (D). The experimental transients and example simulation plots were grouped by the rise time, with the rise time centered about $330 \mu \mathrm{s}(\mathrm{C})$ and $500 \mu \mathrm{s}$ (D). The difference between experiment (colored traces) and the model (black traces) indicates that the hydrodynamic trapping is ultimately overcome, and this can reasonably be attributed to the propulsion of the NP due to the release of oxygen as part of $\mathrm{H}_{2} \mathrm{O}_{2}$ electro-oxidation, as seen at larger "swimmer particles" in solution. ${ }^{30}$ In essence, spatially and temporally nonuniform oxygen generation on the asymmetrical NP (Figure ${ }_{2 B}$ ) after trapping on the collector electrode could accelerate the movement of the $\mathrm{NP}^{31}$ and overcome the hindered diffusion of the NP near the surface, reducing the average occupancy (current) and ultimately leading to the particle moving completely outside the electron transfer region. Further evidence for this mechanism comes from the analysis of responses at $0.75 \mathrm{~V}$ where water oxidation was also initiated, causing higher currents and propulsion effects (Supporting Information, Figure $\mathrm{S}_{5}$ ). The decay time, defined as the time period from $i_{\text {peak }}$ to the time when the current was $10 \%$ of $i_{\text {peak }}$ following the peak was $1.95( \pm 0.15) \mathrm{ms}(N=38)$ compared to $3.08( \pm 0.58) \mathrm{ms}$ at $0.55 \mathrm{~V}(N=29)$, indicating that NPs tend to depart from the electrode quicker at higher bias. Other possible (alternative) reasons for the current-time decay that need to be considered include some deactivation process 9,17 although this is unlikely, first, because such particles are highly active (on average) for long periods when ad- 
sorbed on other electrode surfaces ${ }^{15}$ and, second, due to the subsequent electrochemical events observed that involve the same NP (vide infra). Moreover, the low concentration of $\mathrm{H}_{2} \mathrm{O}_{2}$ used is insufficient for the NP surface to become supersaturated with $\mathrm{O}_{2}$ and for all of the sites on the NP to be deactivated. ${ }^{2}$

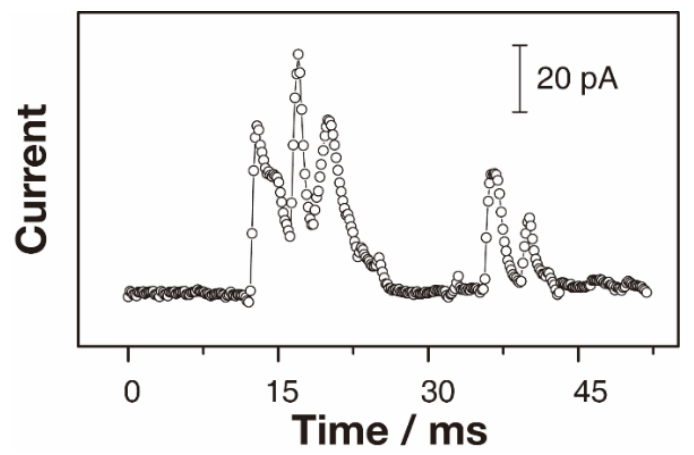

Figure 5. Typical multiple $\mathrm{RuO}_{\mathrm{x}} \mathrm{NP}$ impact events at a collector electrode potential of $0.55 \mathrm{~V}$.

As well as explaining the time scale of the observed current transients, the mechanism of hydrodynamic trapping and release also accounts for the high frequency of NP impacts $\left(f_{\mathrm{NP}}\right)$ observed. Hitherto, a model based on a diffusionlimited flux of NPs at an UME is often used to analyze $f_{\mathrm{NP}}$, given by:4,5

$$
f_{\mathrm{NP}}=4 D_{\mathrm{NP}} C_{\mathrm{NP}} N_{\mathrm{A}} r_{\text {disc }}(2)
$$

where $D_{\mathrm{NP}}$ is the diffusion coefficient of NPs $\left(8.8 \times 10^{-8} \mathrm{~cm}^{2}\right.$ $\left.\mathrm{s}^{-1}\right), C_{\mathrm{NP}}$ is the concentration of NPs $(15 \mathrm{pM}), N_{\mathrm{A}}$ is the Avogadro constant $\left(6.022 \times 10^{23} \mathrm{~mol}^{-1}\right)$, and $r_{\text {disc }}$ is the radius of the electrode (1.5 $\mu \mathrm{m}$ herein). For SECCM the diffusional flux is about $10 \%$ of that for the same sized disc electrode 33 and for the experimental conditions herein, this yields a value of 0.05 $\mathrm{s}^{-1}$. This corresponds to a single pass collision with the collector electrode every $20 \mathrm{~s}$ or so on average. The $f_{\mathrm{NP}}$ we measure is $86 \mathrm{~s}^{-1}$, about 1700-times greater than the $f_{\mathrm{NP}}$ value expected based purely on single-pass diffusion. The reason for the much larger $f_{\mathrm{NP}}$ value is that once the NP has moved away from the electrode and the electrochemical reactions switches off as a consequence, the NP will tend to come back to the electrode surface (hydrodynamic trapping), resulting in another current transient such as in the case shown in Figure 5, where multiple, rapid events are observed. The rapidity of such discrete events, would be very difficult to discern with previously employed set ups. Indeed, some previous studies have acknowledged that the accuracy of the $f_{\mathrm{NP}}$ measurement was low due to instrumental limitations necessitating a slow data acquisition rate. 34 Other studies have reported that the value of $f_{\mathrm{NP}}$ was reasonably similar to that expected for a diffusional flux of NPs. 9 However, even without electrochemically-driven propulsion, it is important to point out that the random walk simulations with hydrodynamic trapping evidence a stochastic interaction of the NP with the collector electrode (simulation traces in Figure ${ }_{4} \mathrm{C}$ and ${ }_{4} \mathrm{D}$; and Supporting Information, Figure S6). Consequently, multiple current events in quick succession are expected for NPs that do not remain affixed (and accumulate) on the electrode surface.

In conclusion, experiments in the SECCM system have provided profound new insights into the interaction of a NP with an electrode surface during impact, using $\mathrm{H}_{2} \mathrm{O}_{2}$ oxidation at $\mathrm{RuO}_{\mathrm{x}} \mathrm{NPs}$ as an illustrative case. The rise time of current-time transients is consistent with random walk simulations for the diffusion of a NP, but with a bias due to hydrodynamic trapping near the electrode due to a greatly reduced diffusion-coefficient. Multiple, rapid current-time curves with very high frequency indicate successive trapping and release of a single NP, with release proposed to be aided by electrochemical propulsion which switches on when a NP hits the electrode, but off again upon NP departure. For the electrochemical fluxes herein, the propulsion is insufficient to completely release the NP at the first attempt and, on average, our measurements show that about 1700 attempts are needed for complete release and loss of the NP from the electrode.

\section{ASSOCIATED CONTENT}

\section{Supporting Information}

Full experimental details, 3D random walk simulations, and supporting $\mathrm{RuO}_{\mathrm{x}} \mathrm{NPs}$ landing experiment data. This material is available free of charge via the Internet at http://pubs.acs.org.

\section{AUTHOR INFORMATION}

\section{Corresponding Author}

*p.r.unwin@warwick.ac.uk.

\section{Notes}

The authors declare no competing financial interests.

\section{ACKNOWLEDGMENTS}

We thank the European Research Council (ERC-20o9-AdG 247143-QUANTIF) for support. This work was also supported by a University of Warwick Chancellor's International Scholarship to M. Kang, a Basic Science Research Program through the National Research Foundation of Korea (2012R1A6A3A03039226) for Y.-R. Kim and EPSRC for a PhD studentship through the MOAC Doctoral Training Centre (EP/F500378/1) for D. Perry.

\section{REFERENCES}

(1) Cox, J. T.; Zhang, B. Annu. Rev. Anal. Chem. 2012, 5, 253.

(2) Sambur, J. B.; Chen, P. Annu. Rev. Phys. Chem. 2014, 65, 395.

(3) Zhang, B.; Fan, L.; Zhong, H.; Lui, Y.; Chen, S. J. Am. Chem. Soc. 2013, 135, 10073.

(4) Xiao, X.; Bard, A. J. J. Am. Chem. Soc. 2007, 129, 9610.

(5) Kwon, S. J.; Zhou, H.; Fan, F-R. F.; Vorobyev, V.; Zhang, B.; Bard, A. J. Phys. Chem. Chem. Phys. 2011, 13, 5394.

(6) Fosdick, S. E.; Anderson, M. J.; Nettleton, E. G.; Crooks, R. M. J. Am. Chem. Soc. 2013, 135, 5994.

(7) Fasari, R.; Robinson, D. A.; Stevenson, K. J. J. Am. Chem. Soc. 2013, 135, 570.

(8) Kim, B.-K.; Kim, J.; Bard, A. J. J. Am. Chem. Soc. 2015, 137, 2343.

(9) Jung, A. R.; Lee, S.; Joo, J. W.; Shin, C.; Bae, H.; Moon, S. G.; Kwon, S. J. J. Am. Chem. Soc. 2015, 137, 1762.

(10) Guo, Z.; Percival, S. J.; Zhang, B. J. Am. Chem. Soc. 2014, 136, 8879 .

(11) Stuart, E. J. E.; Tschulik, K.; Batchelor-McAuley, C.; Compton, R. G. ACS Nano 2014, 8, 7648 .

(12) Wakerley, D.; Güell, A. G.; Hutton, L. A.; Miller, T. S.; Bard, A. J.; Macpherson, J. V. Chem. Commun. 2013, 49, 5657. 
(13) Klejin, S. E. F.; Lai, S. C. S.; Miller, T. S.; Yanson, A. I.; Koper, M. T. M.; Unwin, P. R. J. Am. Chem. Soc. 2012, 134, 18558

(14) Chen, W.; Cai, S.; Ren, Q.; Wen, W.; Zhao, Y. Analyst 2012, 137, 49.

(15) Kang, M.; Lee, Y.; Jung, H.; Shim, J. H.; Lee, N.; Baik, J. M.; Lee, S C.; Lee, C.; Lee, Y.; Kim, M. H. Anal. Chem. 2012, 84, 9485.

(16) Kim, S-J.; Jung, H.; Lee, C.; Kim, M. H.; Lee, Y. Sesor. Actuat. BChem. 2014, 191, 298.

(17) Sardesai, N. P.; Andreescu, D.; Andreescu, S. J. Am. Chem. Soc. 2013, 135, 16770.

(18) Patel, A. N.; Collignon, M. G.; O'Connell, M. A.; Hung, W. O. Y.; McKelvey, K.; Macpherson, J. V.; Unwin, P. R. J. Am. Chem. Soc. 2012 134, 20117.

(19) Patel, A. N.; Tan, S.; Miller, T. S.; Macpherson J. V.; Unwin, P. R. Anal. Chem., 2013, 85, 11755.

(20) S. C. S. Lai, R. A. Lazenby, P. M. Kirkman, P. R. Unwin, Chem. Sci. 2015, 6, 1126

(21) Yang, D.-Q.; Sacher, E. Chem. Mater. 20o6, 18, 1811.

(22) Kleijn, S. E. F.; Lai, S. C. S.; Koper, M. T. M.; Unwin, P. R. Angew. Chem. Int. Ed. 2014, 53, 2.

(23) Jiang, J.; Oberdörster, G.; Biswas, P. J. Nanopart. Res. 2009, 11, 77.
(24) Lee, Y.; Suntivich, J.; May, K. J.; Perry, E. E.; Shao-Horn, Y. J. Phys. Chem. Lett. 2012, 3, 399.

(25) Bard, A. J.; Faulkner, L. R. Electrochemical Methods: Fundamentals and Applications, 2nd ed.; John Wiley \& Sons: New York, 2001.

(26) Bobbert, P. A.; Wind, M.; Vlieger, M. J. Phys. A 1987, 141, 58.

(27) Evans, S. A. G.; Elliott, J. M.; Andrews, L. M.; Bartlett, P. N.; Doyle, P. J.; Denuault, G. Anal. Chem. 2002, 74, 1322.

(28) Zhao, J.; Bradbury, C. R.; Huclova, S.; Potapova, I.; Carrara, M.; Fermin, D. J. J Phys Chem. B 2005, 109, 22985.

(29) Bevan, M. A.; Prieve, D. C. J. Chem. Phys. 2ooo, 113, 1228.

(30) Wang, Y.; Hernandez, R. M.; Bartlett, Jr., D. J.; Bingham, J. M.; Kline, T. R.; Sen, A.; Mallouk T. E. Langmuir, 2006, 22, 10451.

(31) Howse, H. R.; Jones, R. A. L.; Ryan, A. J.; Gough, T.; Vafabakhsh, R.; Golestanian, R., Phys. Rev. Lett. 2007, 99, 048102.

(32) Luo, L.; White, H. S. Langmuir, 2013, 29, 11169.

(33) Snowden, M. E.; Güell, A. G.; Lai, S. C. S.; McKelvey, K.; Ebejer, N.; O'Connell, M. A.; Colburn, A. W.; Unwin, P. R. Anal. Chem. 2012, 84,2483

(34) Kwon, S. J.; Fan, F-R. F.; Bard, A. J. J. Am. Chem. Soc. 2010, 132, 13165 . 


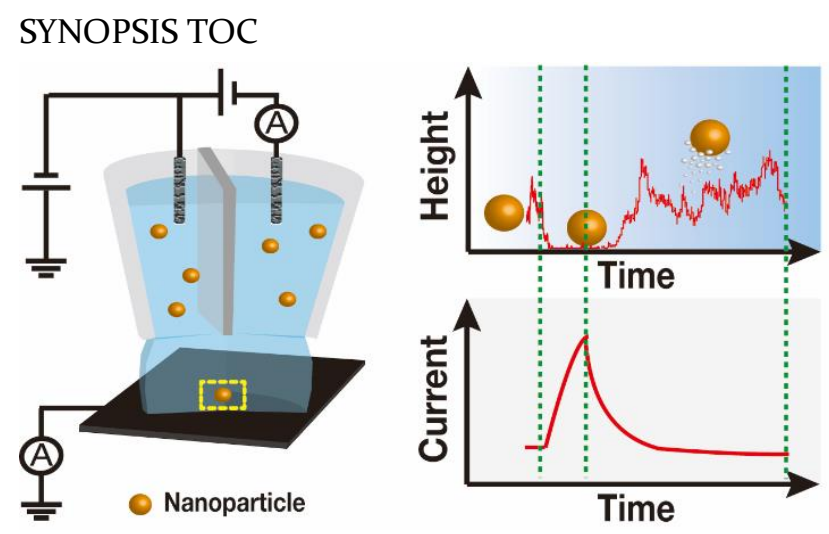

